\section{SAT0236 CLINICAL AND LABORATORY FEATURES OF PATIENTS WITH SJOGREN'S SYNDROME COMPLICATED WITH MALT LYMPHOMA}

A. Torgashina ${ }^{1}$, S. Palshina ${ }^{2}$, E. Sokol ${ }^{2}$, J. Khvan ${ }^{2}$, B. Chaltcev ${ }^{2}$, V. Vasiliev ${ }^{3}{ }^{1}$ V.A. Nasonova Research Institute of Rheumatology, Moscow, Russian Federation; ${ }^{2}$ V.A. Nasonova Research Institute of Rheumatology, Moscow, Russian Federation; ${ }^{3} \mathrm{MEDSI}$, Moscow, Russian Federation

Background: The risk of developing lymphomas in Sjogren's syndrome (SS) is more than 10-fold higher than in general population. Available publications describe a number of indicators considered as predictors for SS development.

Objectives: To describe clinical and laboratory characteristics of patients with SS and MALT Iymphoma and compare them with idem parameters in the control group. To identify factors associated with development of lymphoma.

Methods: The study included 87 SS patients with MALT lymphoma. In all cases lymphoma involved the parotid salivary glands. In all cases MALT lymphoma was diagnosed simultaneously with SS. At the time of inclusion non of the patients was on immunosuppressive therapy. Fifty five SS patients without lymphoproliferative pathology composed the control group. All cases were newly diagnosed and treatment-naïve.

SS was diagnosed based on the ACR-EULAR criteria. The histologic and immunohistochemical diagnosis of lymphoma was performed with B-cell clonality determination in salivary gland tissue.

The following clinical and laboratory parameters were monitored in both groups: rates of stage 3 xerostomia $(<0.5 \mathrm{ml} / 5 \mathrm{~min})$, grade 3 hypolacrimia $(<5 \mathrm{~mm} / 5 \mathrm{~min})$, lymphadenopathy, hemorrhagic rashes, decreased C3, C4 complement components, increased RF, high anti-Ro and anti-La antibodies positivity, hematological changes, serum levels of secretory monoclonal antibodies, cryoglobulinemia, etc. Pearson's $\times 2$ criterion (analysis of contingency tables) was used for statistical analysis. The differences were considered statistically significant when $p$ values were $<0.05$. Statistica 10 for Windows (StatSoft Inc., USA) package was used for statistical data processing.

Results: In the study group 84 patients were females, their mean age at the onset of lymphoma was $53 \pm 10$ years. The disease duration was 7 years (3-12) before the diagnosis was established. All patients in the control group were females, the mean age at diagnosis was $50.2 \pm 13$ years. Patients' age at diagnosis did not differ significantly between the groups. Enlarged salivary or lacrimal glands were found in all SS-MALT patients and in $18 \%$ of patients in the control group.

The rates of such systemic manifestations as polyneuropathy, kidney and joint damage was low and did not differ between groups.

Increased levels of anti-Ro antibodies was documented in the majority of patients in both groups ( $87 \%$ and $81 \%, p=0.4$ ), while aLa antibodies were significantly more common in MALT lymphoma patients $(60 \%$ and $40.3 \%, p=0.045)$.

Similar rates of increased IgG and IgM levels were found in both groups, while increased IgA levels were 6-fold more common in the lymphoma group $(p<0.00001)$. Anemia and leukopenia were documented in approximately $25 \%$, and thrombocytopenia - in $2 \%$ of patients in both groups. Cryoglobulinemia $36 \%$ vs. $24 \%$ ) and circulation of secretory monoclonal immunoglobulins (32\% vs. $18 \%$ ) were more common in the lymphoma group, but the difference was insignificant $(p=0.2)$. The incidence of other clinical and laboratory abnormalities in SS and SS-MALT patients is presented on the graph Forest plot with $\mathrm{OR}$ and $\mathrm{Cl}$ indication.

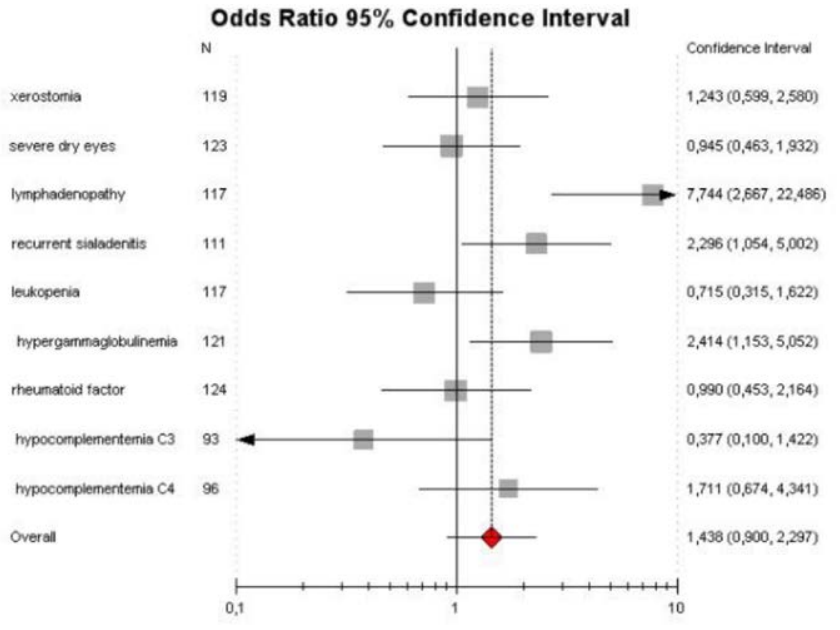

Graphic 1. Clinical and laboratory features of SS patients with MALT-lymphoma compared with SS patients without lymphoma.
Conclusion: Therefore, universally recognized predictors of lymphoma development, such as cryoglobulinemia and hypocomplementemia did not show reliable association with lymphoma. In analyzed cohort development of MALT Iymphoma was statistically significantly associated with recurrent parotitis in past medical history, presence of lymphadenopathy at diagnostic examination, increased levels of anti-La antibodies and $\lg \mathrm{A}$, and hypergammaglobulinemia. Probably we should more actively treat patients with these clinical and laboratory features in order to prevent the development of lymphoma in them.

Disclosure of Interests: None declared

DOI: 10.1136/annrheumdis-2020-eular.5681

\section{\begin{tabular}{|l|l}
\hline SAT0237 THE SYSTEMIC LUPUS INTERNATIONAL & THE
\end{tabular} COLLABORATING CLINICS (SLICC) FRAILTY INDEX (SLICC-FI) PREDICTS DAMAGE ACCRUAL IN SYSTEMIC LUPUS ERYTHEMATOSUS (SLE) PATIENTS. DATA FROM A LATIN AMERICAN MESTIZO COHORT}

M. F. Ugarte-Gil ${ }^{1,2}$, R. V. Gamboa Cárdenas ${ }^{1}$, C. Reategui Sokolova ${ }^{1,3}$, V. Pimentel-Quiroz ${ }^{1,2}$, M. Medina Chinchon ${ }^{1}$, C. Elera-Fitzcarrald ${ }^{1,2}$, J. Alfaro Lozano $^{1}$, Z. Rodriguez Bellido ${ }^{1,4}$, C. Pastor Asurza ${ }^{1,4}$, R. Perich Campos ${ }^{1,4}$ G. S. Alarcon ${ }^{5,6}{ }^{1}$ Hospital Guillermo Almenara Irigoyen, EsSalud, Lima, Peru; ${ }^{2}$ Universidad Científica del Sur, Lima, Peru; ${ }^{3}$ Universidad San Ignacio de Loyola, Unidad de Investigación para la Generación y Síntesis de Evidencias en Salud, Lima, Peru; ${ }^{4}$ Universidad Nacional Mayor de San Marcos, Lima, Peru; ${ }^{5}$ University of Alabama at Birmingham, Birmingham, United States of America; ${ }^{6}$ Universidad Peruana Cayetano Heredia, Lima, Peru

Background: The Systemic Lupus International Collaborating Clinics (SLICC) Frailty Index (SLICC-FI) has been developed as a predictor of outcomes in SLE patients ${ }^{1-3}$. However, it needs to be validated in several populations.

Objectives: To evaluate the SLICC-FI as a predictor of future damage accrual in systemic lupus erythematosus (SLE) patients.

Methods: Patients from a single-center prevalent cohort were included. Damage accrual was defined as the increase in SLICC/American College of Rheumatology (ACR) damage index (SDI) scores between the baseline and last visits. The SLICC-FI was measured at baseline. Univariable and multivariable negative binomial regression were performed to determine the association between the baseline SLICC-FI (per 0.05 increase) and damage accrual during follow-up, adjusted for sex, age at diagnosis, socioeconomic status, disease duration, SLE Disease Activity Index 2000 (SLEDAI-2K), SDI, prednisone daily dose, antimalarial and immunosuppressive drug use at baseline, and duration of follow-up.

Results: Of the 265 patients included, 248 (93.6\%) were female with mean (SD) age 35.1 (13.6) years at diagnosis. At baseline, mean (SD) SLE disease duration was 7.3 (6.5) years, SDI was 1.1 (1.3) and SLEDAI-2K was 5.3 (4.6). The mean (SD) baseline SLICC-FI was $0.22(0.05)$. After a mean (SD) of $5.2(2.2)$ years of follow-up, the SDI increased in $126(47.5 \%)$ patients, and the final mean (SD) SDI score was 1.7 (1.7). Higher SLICC-FI scores at baseline predicted greater damage accrual in the univariable analysis [Incidence Rate Ratio (IRR)=1.283, (CI95\% 1.072-1.536); $p=0.007$ ]. The SLICC-FI remained associated with damage accrual in the multivariable model, after adjustment for possible confounders [IRR $=1.224(\mathrm{Cl} 195 \%$ 1.007-1.488); $\mathrm{p}=0.042]$.

Conclusion: The SLICC-FI predicts damage accrual in prevalent SLE, supporting the relevance of this index in the evaluation of SLE patients. This is the first study validating the SLICC-FI in South American population

References:

[1] Legge A, Kirkland S, Rockwood K, et al. Construction of a Frailty Index as a Novel Health Measure in Systemic Lupus Erythematosus. J Rheumatol. 2020; 47: 72-81

[2] Legge A, Kirkland S, Rockwood K, et al. Evaluating the Properties of a Frailty Index and Its Association With Mortality Risk Among Patients With Systemic Lupus Erythematosus. Arthritis Rheumatol. 2019; 71 1297-107

[3] Legge A, Kirkland S, Rockwood K, et al. Prediction of Damage Accrual in Systemic Lupus Erythematosus Using the Systemic Lupus International Collaborating Clinics Frailty Index (SLICC-FI). Arthitis Rheumatol. Epub ahead of print 2019 Oct 21.

Disclosure of Interests: Manuel F. Ugarte-Gil Grant/research support from: Jannsen, Pfizer, Rocío Violeta Gamboa Cárdenas Grant/research support from: Pfizer, Cristina Reategui Sokolova: None declared, Victor Pimentel-Quiroz: None declared, Mariela Medina Chinchon: None declared, Claudia Elera-Fitzcarrald Consultant of: Tecnofarma, Jose Alfaro Lozano Speakers bureau: Lilly, Zoila Rodriguez Bellido: None declared, Cesar Pastor Asurza: None declared, Risto Perich 